\title{
Duration of wrist immobilization is associated with shoulder pain in patients with after wrist immobilization: an observational study
}

\author{
Raquel Cantero-Téllez', Santiago García Orza² ${ }^{2}$ Mark D. Bishop³ ${ }^{3}$ Pedro Berjano ${ }^{4}$, Jorge Hugo Villafañe ${ }^{5, *}$ \\ 'Physiotherapy Department, Faculty of Health Sciences, University of Malaga, Málaga, Spain \\ ${ }^{2}$ Hospital Comarcal de la Axarquía, Málaga, Spain \\ ${ }^{3}$ Department of Physical Therapy, University of Florida, Gainesville, FL, USA \\ ${ }^{4}$ IRCCS Istituto Ortopedico Galeazzi, Milan, Italy \\ ${ }^{5}$ IRCCS Fondazione Don Carlo Gnocchi, Milan, Italy
}

The main goal of this study was to determinate the extent of the relationship between shoulder pain and time of wrist and thumb immobilization required after injury. One hundred twenty-three consecutive subjects presenting to the practice of different Orthopedic Specialist hospitals with a diagnosis of distal radius or scaphoid fracture that required wrist and thumb immobilization were screened for eligibility criteria. Upper extremity pain and the need for shoulder rehabilitation were assessed using the visual analogue scale (VAS) at baseline after immobilization period (TO) 1- and 3-month follow-up (1-FU/2-FU). More than $35 \%$ patients had shoulder pain (VAS $>40 \mathrm{~mm}$ ) after immobilization of the wrist. Shoulder pain intensity after immobilization and at follow-up (1 and 3 months) was strongly correlated with the duration of the immobilization. Immobilization for 3.5 weeks or longer was the strongest predictor for the need of subsequent shoulder rehabilitation. An increased the time of immobilization of the wrist is associated with an increase in shoulder pain and need for shoulder rehabilitation in patients after wrist fracture.

Keywords: Immobilization, Pain, Shoulder, Wrist

\section{INTRODUCTION}

Shoulder pain is a highly prevalent musculoskeletal problem that often has a multifactorial underlying pathology and is associated with high societal cost. Self-reported prevalence of shoulder pain is estimated to be between 16\% and 26\% (Meislin et al., 2005; Mitchell et al., 2005; Villafañe et al., 2015). The four most common causes of shoulder pain described in the literature include rotator cuff disorders, glenohumeral disorders, acromioclavicular joint disease, and referred neck pain (Bau et al., 2017; Pillastrini et al., 2016).

The joints of the upper extremity are linked in a functional chain in which the function at one joint impacts function at the others. Reaching, for example, requires complex and simultaneous control of movements at the shoulder, elbow and wrist (Liu et al., 2013). In fact, simple finger motions elicit postural changes throughout the entire upper extremity (Caronni and Cavallari, 2009). This limb wide activation occurs to provide proximal stability counteracting torques created distally by two joint muscles crossing the wrist and elbow, for example, presumably to stabilize the shoulder. Limitations at one joint in this system will affect movement at another joint during functional activity. This was demonstrated by who during a simple upper extremity task.

In addition, muscle length decreases when immobilized or mobilized at a shorter length. A shortened muscle will also demonstrate increased passive tension potentially limiting full joint motion; in this case at the wrist and hand (Page, 2012). Due to observed muscle adaptations and changes in interjoint coordination
${ }^{*}$ Corresponding author: Jorge Hugo Villafañe

(ii) https://orcid.org/0000-0002-3239-7626

IRCCS Fondazione Don Carlo Gnocchi, Piazzale Morandi, 6, Milan, Italy

Tel: +39-3395857563, Fax: +39-0307245282, E-mail: mail@villafane.it

Received: June 8, 2018 / Accepted: July 16, 2018
This is an Open Access article distributed under the terms of the Creative Commons Attribution Non-Commercial License (http://creativecommons.org/licenses/by-nc/4.0/) which permits unrestricted non-commercial use, distribution, and reproduction in any medium, provided the original work is properly cited. 
reported in the literature, we hypothesized that as wrist immobilization limits basic activities of daily living (ADLs) and disrupts the interactions of different upper limb muscles, faulty movement patterns may develop resulting in pain, guarding, overuse, or fatigue (Borboni et al., 2017; Cantero-Téllez et al., 2018a; Cantero-Téllez et al., 2018b). Specifically we hypothesized that the duration of immobilization would be positively associated with any reported shoulder pain. Therefore, the primary goal of this study was to establish the extent of the relationship between wrist/thumb immobilization after fracture and shoulder dysfunction. Specifically, we hypothesized that the duration of immobilization would be related to shoulder pain intensity. Next we sought to determine the (a) need for rehabilitation of shoulder dysfunction, and (b) if any relationship existed between the duration of immobilization and rehabilitation.

\section{MATERIALS AND METHODS}

\section{Participants}

A longitudinal, observational study was performed. The study was approved by the local University ethic committee (reference number: CEUMA: 35-2016-H). The participant pool consisted of consecutive patients presenting to the practice of Orthopedic Specialist hospitals from Málaga between April 2015 to October 2016. All participants completed initial screening to confirm wrist and thumb immobilization up to $2 / 3$ proximal to the forearm after distal radius fracture (DRF) or scaphoid fracture. The participation of all subjects was voluntary, and no incentives were given to encourage enrollment. Inclusion criteria for this study were patients 18 years or older, diagnosis of DRF or scaphoid fracture without previous ipsilateral shoulder surgical intervention, wrist or hand associated pathologies or previous history (in the last 2 years) of upper limb fractures, instability, shoulder pain and adhesive capsulitis. Participants with acute prolapsed disc and degenerative neurological illnesses, or major psychiatric condition were excluded. We also excluded patients with wrist fractures that required elbow immobilization. All subjects were right-hand dominant as defined by the hand that they wrote with and gave informed written consent.

\section{Study protocol}

All participants that required wrist and thumb immobilization ( 3 and 8 weeks, respectively) and had no shoulder pain were sent to the hand therapy center after the immobilization period for the first evaluation. A hand therapist took baseline data regarding age, sex, diagnosis, time of immobilization and presence of ipsilateral shoulder pain during ADL using a visual analogue scale (VAS). VAS scale is a unidimensional measure of pain intensity that has been widely used in diverse adult populations, including those with rheumatic diseases (Huskisson, 1974). Sensitivity and reliability were well defined by Breivik et al. (2000) and Lundeberg et al. (2001). In patients with chronic inflammatory or degenerative joint pain, the VAS has demonstrated sensitivity to changes in pain (Villafañe, 2018). Patients were scheduled at one and 3 months for follow-up. All patients received wrist rehabilitation according their pathology, but no shoulder treatment was indicated during this period. The STROBE (Strengthening the Reporting of Observational Studies in Epidemiology) statement checklist of items of cohort observational studies was fulfilled in this article (von Elm et al., 2008).

\section{Statistical analysis}

Data were analyzed using IBM SPSS Statistics ver. 22.0 (IBM Co., Armonk, NY, USA). Descriptive statistics (mean and standard deviation) were provided for all subjects. The relationships between the duration of immobilization and shoulder pain intensity, and subsequent shoulder treatment due to shoulder pain or restriction of mobility variables were assessed using Pearson coefficients.

Multivariate linear regression analyses were performed to evaluate the association of duration of immobilization with pain intensity and diagnosis. The area under the receiver operating characteristic (ROC) curve was calculated, and the sensitivity and specificity of the VAS and duration of immobilization ratio at T0, 1-FU (1 month), and 2-FU (3 months) were calculated for the entire sample. The statistical analysis was conducted at a $95 \%$ confidence level, and a $P<0.05$ was considered statistically significant.

\section{RESULTS}

One hundred twenty-three consecutive subjects with DRF or scaphoid fracture that required wrist and thumb immobilization were screened for eligibility criteria. Thirty-one patients were excluded due to shoulder or persistent neck pain in the last year $(\mathrm{n}=16)$, medial nerve compression $(\mathrm{n}=4)$, cubital nerve lesion $(\mathrm{n}=2)$ and previous wrist or shoulder surgical intervention $(\mathrm{n}=9)$. Ninety-two participants, satisfying all eligibility criteria, agreed to participate. The mean age of the sample was $42.0 \pm 14.7$ years, with $47.8 \%$ women, and the mean duration of immobilization wrist was $4.8 \pm 2.0$ weeks (Table 1 ). 
Table 1. Baseline demographics $(n=92)$

\begin{tabular}{lc}
\hline Variable & Value \\
\hline Age (yr) & $42.0 \pm 14.7$ \\
Sex & \\
Male & $48(52.2)$ \\
Female & $44(47.8)$ \\
Diagnosis & \\
Scaphoid fracture & $38(41.3)$ \\
Distal Radius fracture & $54(58.7)$ \\
\hline
\end{tabular}

Values are presented as mean \pm standard deviation or number (\%).

Table 2. Pearson correlation coefficient between shoulder pain and time of immobilization

\begin{tabular}{lccccc}
\hline VAS & Value & $\begin{array}{c}\text { Time of } \\
\text { immobilization } \\
(\text { wk })\end{array}$ & $\begin{array}{c}\text { Scaphoid } \\
\text { fracture }\end{array}$ & $\begin{array}{c}\text { Female } \\
\text { sex }\end{array}$ & $\begin{array}{c}\text { Shoulder } \\
\text { treatments }\end{array}$ \\
\hline VAS.T0 & $r$ & $0.880^{*}$ & $0.878^{*}$ & 0.072 & $0.793^{*}$ \\
VAS.1-FU & $P$-value & $<0.001$ & $<0.001$ & 0.498 & $<0.001$ \\
& $P$-value & $0.891^{*}$ & $0.875^{*}$ & 0.027 & $0.822^{*}$ \\
VAS.2-FU & $r$ & 0.001 & $<0.001$ & 0.798 & $<0.001$ \\
& $P$-value & $<0.001$ & 0.003 & 0.971 & 0.003 \\
\hline
\end{tabular}

VAS, visual analogue scale; TO, after immobilization; 1-FU, 1-month follow-up; 2-FU, 3-month follow-up.

${ }^{*}$ Correlation is significant at the 0.01 level (2-tailed).

Thirty-nine participants (35.9\%) reported shoulder pain after immobilization (VAS.T0 $>40 \mathrm{~mm}$ ). Of these 37 patients had a scaphoid fracture which required a longer period of immobilization. Pain intensity was strongly correlated with the time of immobilization both immediately after immobilization and follow-up period ( 1 and 3 months) $(P<0.001)$ (Table 2$)$.

Twenty-six participants in the study $(66.7 \%$ of patients reporting shoulder pain) required shoulder rehabilitation treatment prescribe by a hand surgeon, due to reduced range of motion (ROM) and/or pain in the shoulder after 3 weeks of wrist immobilization. Twenty-five of these patients had a scaphoid fracture and only one a DRF. Sixteen patients remained with VAS $>40 \mathrm{~mm}$ at 3-month follow-up. The results showed a correlation between the need to receive rehabilitation treatment in the ipsilateral shoulder after wrist injury and the duration of the immobilization $(P<0.001)$.

The optimal cutoff points predicting whether or not a patient needed rehabilitation for the ipsilateral shoulder were approximately 3.5 weeks of immobilization $(97.0 \%$ sensitivity; $32.2 \%$ specificity), $35 \mathrm{~mm}$ for the VAS.T0 ( $97.4 \%$ sensitivity; $3.7 \%$ specificity), $40 \mathrm{~mm}$ for the VAS.1-FU ( $92.1 \%$ sensitivity; $3.7 \%$ specificity) and $15 \mathrm{~mm}$ for the VAS.2-FU (63.2\% sensitivity; $25.9 \%$
Table 3. Area under the receiver operating characteristic curve for each instrument $(n=92)$

\begin{tabular}{lccccc}
\hline Instrument & Area & $95 \% \mathrm{Cl}$ & $\begin{array}{c}\text { Optimal cutoff } \\
\text { value for change } \\
\text { on instrument }\end{array}$ & $\begin{array}{c}\text { Sensitivity } \\
\text { at optimal } \\
\text { cutoff }(\%)\end{array}$ & $\begin{array}{c}\text { Specificity at } \\
\text { optimal } \\
\text { cutoff }(\%)\end{array}$ \\
\hline VAS.T0 & $0.985^{\dagger}$ & $0.96-1.00$ & 35.0 & 97.4 & 3.7 \\
VAS.1-FU & $0.966^{\dagger}$ & $0.93-1.00$ & 40.0 & 92.1 & 3.7 \\
VAS.2-FU & $0.669^{*}$ & $0.56-0.78$ & 15.0 & 63.2 & 25.9 \\
Weeks & $0.963^{\dagger}$ & $0.92-0.99$ & 3.5 & 97.0 & 32.0 \\
Age & 0.480 & $0.36-0.61$ & 37.0 & 57.9 & 55.6 \\
\hline
\end{tabular}

$\mathrm{Cl}$, confidence interval; VAS, visual analogue scale; T0, after immobilization; 1-FU, 1-month follow-up; 2-FU, 3-month follow-up.

${ }^{*}$ Correlation is significant at the 0.05 level. ${ }^{+}$Correlation is significant at the 0.01 level.

specificity). The area under the ROC curve for the duration of immobiliza-tion was 0.963 (95\% confidence interval, 0.92-0.99) (Table 3).

\section{DISCUSSION}

It's known that long immobilization periods of wrist joint lead to overall complication rates at the wrist ranging from $6 \%$ to $80 \%$. These complications not only include complex regional pain syndrome, stiffness, perineal wrist nerve injury, tendon and ligament injuries, but wrist reductions in ROM, muscular atrophy, and loss of movement representation (Diaz-Garcia et al., 2011). The purpose of the current study was to determine if wrist and thumb immobilization after DRF or scaphoid fracture could also cause proximal complications such as shoulder pain. Our primary finding was a strong positive correlation between duration of immobilization and shoulder pain intensity both at the first and third month after cast removal.

Kuo et al. (2013) suggested that early rehabilitative intervention for DRF treatment, avoid additional complications. This results regarding the benefit of reducing immobilization, are in concordance with Schott and Korbus (2014) who describe a 20\% of patients reporting persistent symptoms after DRF, and ongoing difficulties and dysfunction after the typical recovery period. However, none of these studies determined the possible causes or intensity of symptoms that prolong patient's recovery time. According to our results, the intensity of shoulder pain was related to the period of wrist immobilization and not to the injury itself. Additionally, our data show that immobilization of 3.5 weeks or longer was very strongly associated with the need for rehabilitation for the shoulder, a joint unaffected by the original injury at the wrist. 
We posit that an increase the increased shoulder pain intensity after wrist immobilization could be due to two primary factors. First, compensatory proximal movements during upper extremity activity are likely if the wrist is unable to performing functions related to positioning the hand. Potentially larger amplitude movements than normal may stress the connective and contractile tissues of the shoulder resulting in pain. A second, and related reason are changes in the internal loading of the shoulder muscles. Previous studies establish a neuromechanical relationship between shoulder and hand; for example, Alenabi et al. (2013) who demonstrated the presence of activity in the shoulder musculature during ipsilateral elbow, wrist, and finger movements. Others as Labriola et al. (2005), Antony and Keir (2010), and Hodder and Keir (2012) found alterations in muscle activity patterns with a redistribution of force in the rotator cuff muscles during gripping, suggesting coactivation of the proximal and distal arm muscles indicating postural activation in the proximal shoulder muscles. It is feasible that changes in grasp that occurs during wrist immobilization, modifies activity of the rotator cuff (Hodder and Keir, 2012). Such a modification may alter glenohumeral mechanics or directly stress the rotator cuff tendons.

Regardless of the primary cause of shoulder pain after wrist immobilization, our findings reinforce the importance of a comprehensive management strategy for the entire upper extremity in response to injury of any component of the functional unit. The necessity of such a program is increased if the patient is likely to be immobilized for longer than 3.5 weeks.

The duration of wrist immobilization strongly correlates with developing ipsilateral shoulder pain that requires treatment. Future electromyography studies and muscle activity pattern should investigate activation of the different muscles groups of the shoulder with or without wrist immobilization with the aim of being able to establish robust preventive shoulder treatments for $\mathrm{pa}$ tients who are likely to be immobilized for longer than 3 weeks after wrist fracture.

\section{CONFLICT OF INTEREST}

No potential conflict of interest relevant to this article was reported.

\section{REFERENCES}

Alenabi T, Jackson M, Tétreault P, Begon M. Electromyographic activity in the immobilized shoulder musculature during ipsilateral elbow, wrist, and finger movements while wearing a shoulder orthosis. J Shoulder Elbow Surg 2013;22:1400-1407.

Antony NT, Keir PJ. Effects of posture, movement and hand load on shoulder muscle activity. J Electromyogr Kinesiol 2010;20:191-198.

Bau JG, Chia T, Wei SH, Li YH, Kuo FC. Correlations of neck/shoulder perfusion characteristics and pain symptoms of the female office workers with sedentary lifestyle. PLoS One 2017;12:e0169318.

Borboni A, Villafañe JH, Mullè C, Valdes K, Faglia R, Taveggia G, Negrini S. Robot-assisted rehabilitation of hand paralysis after stroke reduces wrist edema and pain: a prospective clinical trial. J Manipulative Physiol Ther 2017;40:21-30.

Breivik EK, Björnsson GA, Skovlund E. A comparison of pain rating scales by sampling from clinical trial data. Clin J Pain 2000;16:22-28.

Cantero-Téllez R, Valdes K, Schwartz DA, Medina-Porqueres I, Arias JC, Villafañe JH. Necessity of immobilizing the metacarpophalangeal joint in carpometacarpal osteoarthritis: short-term effect. Hand (N Y) 2018a; 13:412-417.

Cantero-Téllez R, Villafañe JH, Valdes K, Berjano P. Effect of immobilization of metacarpophalangeal joint in thumb carpometacarpal osteoarthritis on pain and function. A quasi-experimental trial. J Hand Ther 2018b;31:68-73.

Caronni A, Cavallari P. Anticipatory postural adjustments stabilise the whole upper-limb prior to a gentle index finger tap. Exp Brain Res 2009; 194:59-66.

Diaz-Garcia RJ, Oda T, Shauver MJ, Chung KC. A systematic review of outcomes and complications of treating unstable distal radius fractures in the elderly. J Hand Surg Am 2011;36:824-835.e2.

Hodder JN, Keir PJ. Targeted gripping reduces shoulder muscle activity and variability. J Electromyogr Kinesiol 2012;22:186-190.

Huskisson EC. Measurement of pain. Lancet 1974;2:1127-1131.

Kuo LC, Yang TH, Hsu YY, Wu PT, Lin CL, Hsu HY, Jou IM. Is progressive early digit mobilization intervention beneficial for patients with external fixation of distal radius fracture? A pilot randomized controlled trial. Clin Rehabil 2013;27:983-993.

Labriola JE, Lee TQ, Debski RE, McMahon PJ. Stability and instability of the glenohumeral joint: the role of shoulder muscles. J Shoulder Elbow Surg 2005;14(1 Suppl S):32S-38S.

Liu W, Whitall J, Kepple TM. Multi-joint coordination of functional arm reaching: induced position analysis. J Appl Biomech 2013;29:235-240.

Lundeberg T, Lund I, Dahlin L, Borg E, Gustafsson C, Sandin L, Rosén A, Kowalski J, Eriksson SV. Reliability and responsiveness of three different pain assessments. J Rehabil Med 2001;33:279-283.

Meislin RJ, Sperling JW, Stitik TP. Persistent shoulder pain: epidemiology, pathophysiology, and diagnosis. Am J Orthop (Belle Mead NJ) 2005;34(12 Suppl):5-9. 
Mitchell C, Adebajo A, Hay E, Carr A. Shoulder pain: diagnosis and management in primary care. BMJ 2005;331:1124-1128.

Page P. Current concepts in muscle stretching for exercise and rehabilitation. Int J Sports Phys Ther 2012;7:109-119.

Pillastrini P, Rocchi G, Deserri D, Foschi P, Mardegan M, Naldi MT, Villafañe JH, Bertozzi L. Effectiveness of neuromuscular taping on painful hemiplegic shoulder: a randomised clinical trial. Disabil Rehabil 2016; 38:1603-1609.

Schott N, Korbus H. Preventing functional loss during immobilization after osteoporotic wrist fractures in elderly patients: a randomized clini- cal trial. BMC Musculoskelet Disord 2014;15:287.

Villafañe JH. Does "time heal all wounds" still have a future in osteoarthritis? Clin Exp Rheumatol 2018;36:513.

Villafañe JH, Valdes K, Anselmi F, Pirali C, Negrini S. The diagnostic accuracy of five tests for diagnosing partial-thickness tears of the supraspinatus tendon: A cohort study. J Hand Ther 2015;28:247-251.

von Elm E, Altman DG, Egger M, Pocock SJ, Gøtzsche PC, Vandenbroucke JP; STROBE Initiative. The strengthening the reporting of observational studies in epidemiology (STROBE) statement: guidelines for reporting observational studies. J Clin Epidemiol 2008;61:344-349. 